\title{
MHD MECHANISMS FOR THE FORMATION OF GALACTIC CEN- TER LOBES
}

\author{
Kazunari SHIBATA \\ Department of Earth Sciences, \\ Aichi University of Education, Japan
}

\begin{abstract}
I critically discuss three possible MHD mechanisms for the formation of Galactic center lobes (GCL) found by Sofue and Handa (1984) from the theoretical point of view. The three mechanisms I shall discuss are: (1) sweeping-magnetic-twist mechanism, (2) explosion in a disk with a vertical magnetic field, and (3) nonlinear Parker instability. I review the characteristics of these mechanisms, which are mainly obtained from nonlinear 2D MHD numerical simulations, and discuss their merits and demerits as possible mechanisms for the formation of GCL and related magnetic structures.
\end{abstract}

\section{Introduction}

Sofue and Handa (1984) found a prominent radio lobe with a size of about $200 \mathrm{pc}$ above the center of our Galaxy. This Galactic Center Lobe (hereafter, referred to as GCL) has an $\Omega$ shape, or a hollow cylindrical shell structure, and seems to emanate from the Galactic center HII gas disk. Sofue and Handa suggested that the GCL may be a low energy prototype of radio jets observed in active galaxies and quasars, and discussed three different models for the GCL: (1) jets driven by magnetic-twist, (2) blast shell due to explosion, and (3) magnetic loop produced by Parker instability.

On the other hand, the vertical branch of the 'radio arc' is located just along the extension of the eastern ridge of the GCL, and shows a significant filamentary structure suggesting some effects of a strong magnetic field (Yusef-Zadeh et al. 1984). This suggestion, especially the presence of strong poloidal magnetic field, was confirmed later by observation of radio polarization (Inoue et al. 1984; Tsuboi et al. 1985).

Here, I will review some attempts to model the GCL and related magnetic structure, such as the radio arc. In these attempts, the three different mechanisms discussed by Sofue and Handa (1984) have been studied using nonlinear twodimensional (2D) MHD numerical simulations.

\section{Sweeping-Magnetic-Twist Mechanism}

Uchida, Shibata and Sofue (1985) proposed an MHD model for the GCL in which the gas in the GCL is lifted up from the HII gas disk by the $\mathbf{J} \times \mathbf{B}$ force in relaxing magnetic twists, which are generated by the interaction of the rotation of the contracting disk with the poloidal magnetic field. This model is based on the MHD model for astrophysical jets developed by Uchida and Shibata, referred to as the 

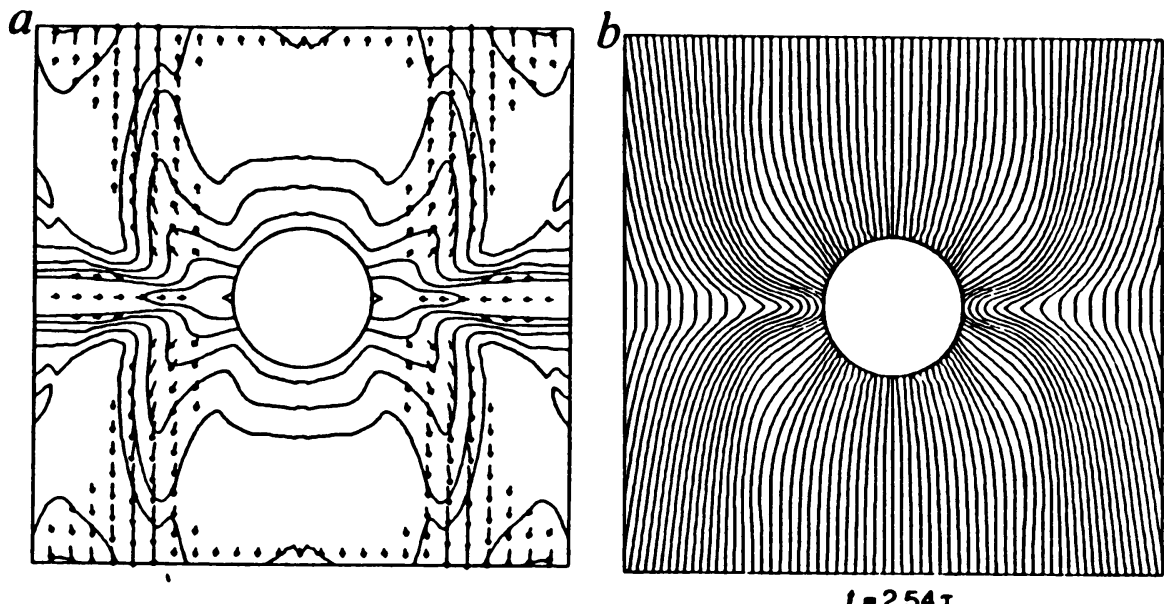

Fig. 1 The sweeping-magnetic-twist model for the GCL (Uchida, Shibata, Sofue 1985). (a) the poloidal velocity vector overlain on the density contour, (b) the poloidal magnetic field lines.

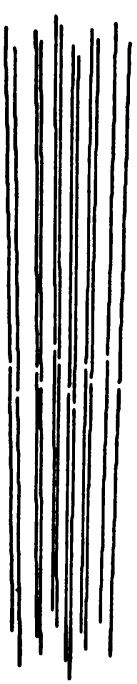

0.00

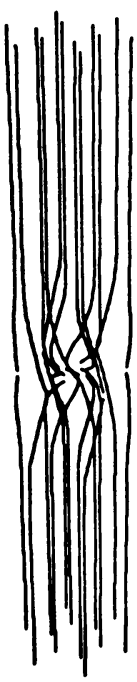

1.22

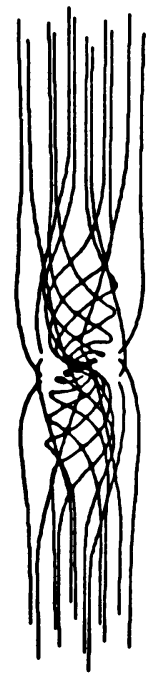

2. 37

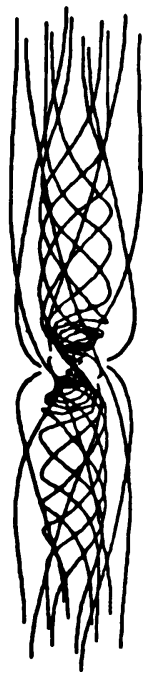

3. 55

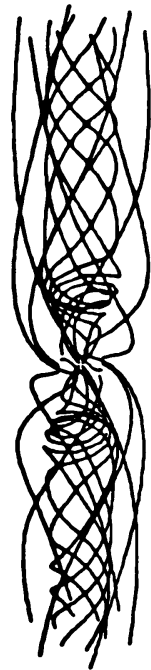

4.66

Fig. 2 The three-dimensional view of magnetic fiel lines in the sweepingmagnetic-twist model for astrophysical jets (Shibata and Uchida 1988, in preparation). 
sweeping-magnetic-twist mechanism (Uchida and Shibata 1985, 1986; Shibata and Uchida 1986, 1987). This is a non-steady mechanism but includes the magneticcentrifugal-wind from the disk (Blandford and Payne 1982; Pudritz and Norman 1983; Lovelace et al. 1987) as a special (steady) version.

The result of their 2D axisymmetric MHD simulation (Fig. 1) agrees well with some basic observational facts about the GCL; cylindrical shell structure of the GCL, and polarization features indicating that the line-of-sight component of the magnetic field reverses direction above and below the Galactic plane. The latter is naturally explained as the helically twisted magnetic field lines which are pulled by the rotation of the disk (Fig. 2).

However, some problems remain. One is the predicted curvature of the poloidal field (hourglass-shaped configuration caused by the accretion of gas). Actual poloidal fields seem to be almost vertical to the Galactic plane. The other concerns the pointmass gravitational potential which Uchida et al. (1985) assumed. In the actual Galactic center region around $r \simeq 10-100 \mathrm{pc}$, the rotation curve is not Keplerian but is nearly flat (e.g. Oort 1977).

Thus, Shibata and Uchida (1987) extended the model by Uchida et al. (1985) to that in a flat-rotation potential (FRP) suitable for the Galactic center region. Their results have shown that the basic mechanism works as well, but the velocity of the jet in FRP is much smaller than that for the point-mass-potential for the same parameter values, although the poloidal magnetic field agrees better with the observed configuration. In order to produce a prominent jet in FRP similar to the GCL, the initial rotational velocity of the disk should be much less than the rotational velocity in the centrifugo-gravitational equilibrium. Since the magnetic braking is not so efficient for $B \leq 40 \mu \mathrm{G}$ to produce such a small rotational velocity, this means that the angular momentum of the disk must be removed by other processes (e.g. gravitational torque) before the disk contracts to the radius of $50-$ $100 \mathrm{pc}$ (Shibata and Uchida 1987).

\section{Explosion in a Disk with a Vertical Magnetic Field}

Umemura et al. (1988) studied the propagation of MHD shock waves through a Galactic nuclear disk $(r \simeq 100 \mathrm{pc}$ ) with a vertical magnetic field. This model is an extended version of the non-magnetic explosion model of Sofue (1984). The model with parameters of $B \simeq 40 \mu \mathrm{G}$ and explosion energy $\simeq 2 \times 10^{53} \mathrm{erg}$ reproduces observed characteristics of the GCL (Fig. 3) such as the nearly vertical field lines in the hollow cylindrical dense shell. Note that the surface of the shell is not the shock front but the contact surface, though the reverse (MHD fast) shock is situated just inside the contact surface. The original (MHD fast) shock propagates farther away from the shell. These morphological structures are similar to those in the MHD model of solar coronal transients (Steinolfson 1985). The origin of the explosion is either the explosive mass ejection from Sgr A or the successive supernova explosions as a result of bursting star formation in the Galactic center region $(r \simeq 50-100 \mathrm{pc})$. 


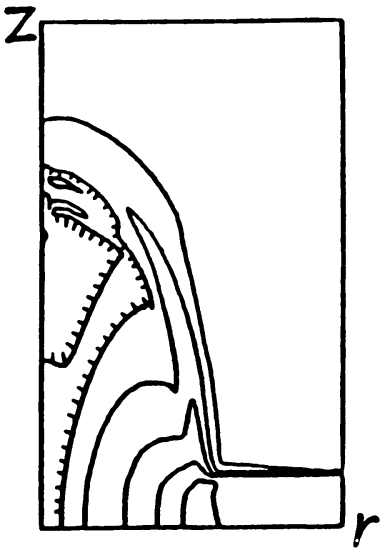

(a)

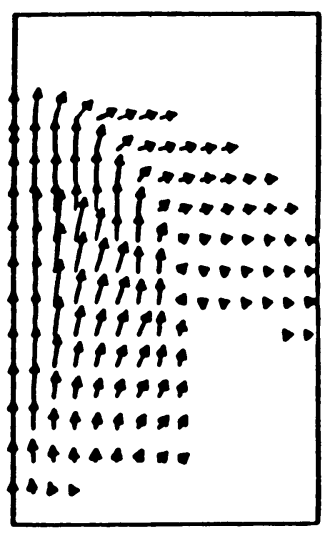

(b)

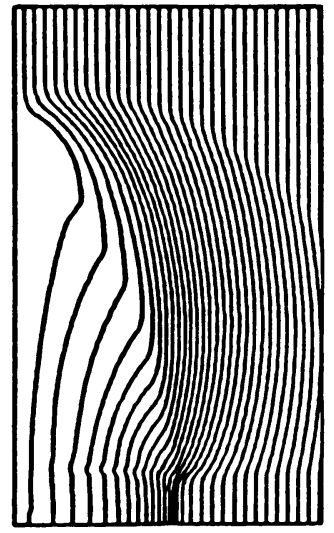

(c)

Fig. 3 The magnetic-explosion model for the GCL (Umemura et al. 1988). (a) density contours, (b) velocity vectors, (c) magnetic field lines.

\section{Nonlinear Parker Instability}

Recently, extending the first 2D MHD numerical simulations of the Parker instability by Matsumoto et al. (1988), Shibata et al. (1989) have studied the nonlinear Parker instability of isolated magnetic flux initially embedded in a field-free disk. According to their results, the nonlinear expansion of the magnetic loop is approximately selfsimilar, and the rise velocity of the loop increases linearly with height as long as the sum of the magnetic and gas pressures outside the disk is much smaller than the magnetic pressure in the loop. By using the appropriate physical parameters in the Galactic center region, the rise velocity of the loop at the height of about 100 $\mathrm{pc}$ is estimated to be about $200 \mathrm{~km} / \mathrm{s}$. Although the morphology of the loop is not very similar to that of the GCL, general features of the simulation may be applied to the GCL and the radio arc, and the velocity obtained above may be compared with observed values in these regions. Note also the analogy with the solar coronal transient: Low (1984) proposed the model where the transients are initiated by Parker (magnetic buoyancy) instability.

\section{Conclusion}

(1) The sweeping-magnetic-twist mechanism explains the basic structure of the GCL and the toroidal magnetic field configuration. However, some problems remain such as the apparent discrepancy between the predicted and observed curvature of the poloidal field, and whether the disk dynamically contracted to $r \simeq 50-100 \mathrm{pc}$ with small initial rotational velocity or not. This mechanism may be better applied to the small-scale (but more energetic) jet emanating from the nucleus ( $\operatorname{Sgr} \mathbf{A}$ ) than to the GCL.

(2) The explosion in a vertical magnetic field reproduces the morphology of both poloidal field and shell in the GCL. The remaining problem is what produced the 
explosion.

(3) At present, the results of numerical simulation of the nonlinear Parker instability do not better reproduce the observed morphology of the GCL than other mechanisms. However, the basic physics of the simulation may be applied to the GCL, in particular to the radio arc.

Finally, it is possible that all three mechanisms work simultaneously in the Galactic center region, which makes phenomena very complex and observational facts difficult to understand. Key observations which distinguish each of the three mechanisms may be observations of the velocity field (rotation around the axis of the jet for the sweeping-magnetic-twist model, downflow along the loop for Parker instability model), and X-ray emission from a hot $\left(\simeq 10^{7} \mathrm{~K}\right)$ gas for the explosion model.

\section{References}

Blandford, R. D. and Payne, D. G. 1982, Mon. Not. Roy. Astron. Soc., 199,883 .

Inoue, M., Takahashi, T., Tabara, H., Kato, T. and Tsuboi, M. 1984, Pub. Astr. Soc. Japan, 36, 633.

Lovelace, R. V. E., Wang, J. C. L. and Sulkanen, M. E. 1987, Ap. J., 315, 504.

Low, B. C. 1984, Ap. J., 281, 392.

Matsumoto, R., Horiuchi, T., Shibata, K. and Hanawa, T. 1988, Pub. Astr. Soc. Japan, 40, 171.

Oort, J. H. 1977, Ann. Rev. Astr. Ap., 15, 295.

Pudritz, R. E. and Norman, C. 1983, Ap. J., 274, 677.

Shibata, K. and Uchida, Y. 1986, Pub. Astr. Soc. Japan, 38, 361.

Shibata, K. and Uchida, Y. 1987, Pub. Astr. Soc. Japan, 39, 559.

Shibata, K., Tajima, T., Matsumoto, R., Horiuchi, T., Hanawa, T., Rosner, R. and Uchida, Y., 1989, Ap. J., Mar. 1 issue.

Sofue, Y. 1984, Pub. Astr. Soc. Japan, 36, 539.

Sofue, Y. and Handa, T. 1984, Nature, 310, 568.

Steinolfson, R. S. 1985, in Collisionless Shocks in the Heliosphere: Reviews of Current Research, American Geophys. U., p. 1.

Tsuboi, M., Inoue, M., Handa, T., Tabara, H., and Kato, T. 1985, Pub. Astr. Soc. Japan, 37, 359.

Uchida, Y. and Shibata, K. 1985, Pub. Astr. Soc. Japan, 37, 515.

Uchida, Y. and Shibata, K. 1986, Can. J. Phys., 64, 507.

Uchida, Y., Shibata, K. and Sofue, Y., 1985, Nature, 317, 699.

Umemura, S., Iki, K., Shibata, K. and Sofue, Y., 1988, Pub. Astr. Soc. Japan, 40, 25.

Yusef-Zadeh, F., Morris, M. and Chance, D. 1984, Nature, 310, 557. 